\title{
An Improved Hybridizing Biogeography-Based Optimization with Differential Evolution for Global Numerical Optimization
}

\author{
Si-ling Feng ${ }^{1,2, a}$, Qing-xin Zhu' ${ }^{1, b}$, Xiu-jun Gong ${ }^{3, c^{*}}$, Sheng Zhong ${ }^{2, d}$ \\ ${ }^{1}$ School of Computer Science \& Engineering, University of Electronic Science and Technology of \\ China, Chengdu, China; \\ ${ }^{2}$ College of Information Science \& Technology, Hainan University, Haikou, China; \\ ${ }^{*}$ School of Computer Science and Technology, Tianjin University, Tianjin, China \\ a fengsiling2008@163.com, ${ }^{\text {b }}$ qxzhu@uestc.edu.cn, ${ }^{c}$ gongxj@tju.edu.cn, ${ }^{d}$ \\ shengzhong62@gmail.com
}

Keywords: Biogeography-Based Optimization; Differential evolution; Global numerical optimization

\begin{abstract}
Biogeography-based optimization (BBO) is a new biogeography inspired algorithm. It mainly uses the biogeography-based migration operator to share the information among solution. Differential evolution (DE) is a fast and robust evolutionary algorithm for global optimization. In this paper, we applied an improved hybridization of BBO with DE approach, namely BBO/DE/GEN, for the global numerical optimization problems. BBO/DE/GEN combines the exploitation of BBO with the exploration of DE effectively and the migration operators of BBO were modified based on number of iteration to improve performance. And hence it can generate the promising candidate solutions. To verify the performance of our proposed BBO/DE/GEN, 6 benchmark functions with a wide range of dimensions and diverse complexities are employed. Experimental results indicate that our approach is effective and efficient. Compared with BBO and $\mathrm{BBO} / \mathrm{DE}$ approaches, $\mathrm{BBO} / \mathrm{DE} / \mathrm{GEN}$ performs better, or at least comparably, in terms of the quality of the final solutions and the convergence rate.
\end{abstract}

\section{Introduction}

Biogeography-based optimization (BBO) algorithm (Simon, 2008) is based on the mechanism of the species migrating from one island to another in nature. Based on two main operators, migration and mutation, $\mathrm{BBO}$ is of good exploitation ability, but it is weak in exploration search. As another comparatively new evolutionary algorithm, differential evolution (DE) is good at exploring global searching space with three control parameters. In order to enhance the exploration ability of BBO, we hybridize the exploitation of BBO with the exploration of $\mathrm{DE}$ to design a hybrid algorithm named $\mathrm{BBO} / \mathrm{DE}$ for global numerical optimization problems.

In this paper, we proposed an improved hybrid $\mathrm{BBO} / \mathrm{DE}$ algorithm, which incorporates the mutation procedure inherited from DE to replace the BBO-based mutation, for global numerical optimization problems. And a new migration operator is proposed based on number of iteration to improve performance. Especially, the migration and mutation mechanism are combine so that the exploration and exploitation abilities are enhanced. Experiments have been tested on 6 benchmark functions, which demonstrate the validity and effectiveness of the proposed improved hybrid algorithm.

The remainder of the paper is organized as follows. Function optimization problem and related implementation technology are described in Section 2. The hybrid algorithm for global numerical optimization problems is proposed in Section 3. Comparisons with some existing approaches are provided in Section 4. Finally, we draw the conclusions in Section 5.

\section{Preliminary}

\subsection{Problem definition}


Global numerical optimization problems are frequently arisen in almost every field of engineering design, applied sciences, molecular biology and other scientific applications. In global numerical optimization problems, the major challenge is that an algorithm may be trapped in the local optima of the objective function. This issue is particularly challenging when the dimension is high. In this work, we only consider the unconstrained function optimization.

\subsection{Differential evolution}

Differential evolution [1] is a simple EA that creates new candidate solutions by combining the parent individual and several other individuals of the same population. A candidate replaces the parent only if it has better fitness. DE's advantages are its simple structure, ease of use, speed and robustness. Due to these advantages, it has been applied to many real world applications, such as data mining, pattern recognition, digital filter design, neural network training, etc. Most recently, DE has also been used for optimal power problem [2].

\subsection{Biogeography-based optimization}

The biogeography-based optimization algorithm proposed by Simon is a new nature-inspired computation technique based on the mathematical models of biogeography to find the optimal solution of the problem [3]. The solution process is analogous to the migration of the species in nature. The BBO algorithm operates on a population of individuals called habitats (or islands). Each habitat represents a possible solution to the problem in hand. The fitness of each habitat is determined by its habitat suitability index (HSI). High HSI solutions are more likely to share their features with other solutions, and low HSI solutions are more likely to accept shared features from other solutions. The sharing of features between solutions is represented as immigration and emigration between the islands [4].

\section{BBO/DE/GEN for global numerical optimization problems}

As mentioned above, BBO has a good exploitation for global optimization [3]. However, it is slow exploring of the search space. On the other hand, DE is good at exploring the search space and locating the region of global minimum. Based on these considerations, in order to balance the exploration and the exploitation of $\mathrm{BBO}$, in this work, we propose a hybrid $\mathrm{BBO}$ approach, called BBO/DE/GEN, which combines the exploitation of BBO with the exploration of DE effectively. And a new migration operator is proposed based on number of iteration to improve performance.

\subsection{Mutation operator for global numerical optimization problems}

In $\mathrm{BBO}$, if a solution is selected for mutation, then it is replaced by a randomly generated new solution set. This random mutation affects the exploration ability of $\mathrm{BBO}$. In BBO/DE/GEN, modified mutation operator creates new feasible solution by inheriting from DE to replace the BBO-based mutation. A mutated individual SIV $\left(H_{i}(j)\right)$ is generated according to the following equation

$$
H_{i}(j)=H_{i}(j)+F^{*}\left(H_{\text {best }}(j)-H_{i}(j)\right)+F^{*}\left(H_{r 1}(j)-H_{r 2}(j)\right)
$$

Where $H_{i}(j)$ is the parent SIV to be mutated, F is the mutation scaling factor. $H_{r_{1}}(j), H_{r_{2}}(j)$ is the randomly selected SIV ( $r \in[1$, popsize $]$ ). In BBO/DE/GEN, This mutation scheme tends to increase the diversity among the population.

\subsection{Migration operator}

In BBO, there are two main operators: migration and mutation. Modified migration operator is a generalization of the standard BBO migration operator and which is motivated from [5]. Modified migration is defined as

$$
H_{i}(s)=\frac{t}{t_{\max }} H_{i}(s)+\left(1-\frac{t}{t_{\max }}\right) H_{j}(s)
$$


Where $H_{i}$ is the ith candidate solution in the BBO population, $\mathrm{s}$ is its solution feature, and $\mathrm{t}$ is number of iteration, $t_{\max }$ is maximum number of iteration. Equation (2) means that a solution feature of solution $H_{i}$ is comprised of two components: the migration of a feature from another solution, and the migration of a feature from itself.

\subsection{Main procedure of improved BBO/DE for global numerical optimization}

By incorporating the above-mentioned migration operator and mutation operator into $\mathrm{BBO}$, the $\mathrm{BBO} / \mathrm{DE} / \mathrm{GEN}$ approach is developed and shown in Algorithm 1. BBO/DE/GEN is able to explore the new search space with the mutation operator of DE and to exploit the population information with the migration operator of $\mathrm{BBO}$. This feature overcomes the lack of exploration of the original $\mathrm{BBO}$ algorithm. Note that the only difference between BBO/DE/GEN and the original BBO algorithm is that $\mathrm{DE}$ is used to replace the original $\mathrm{BBO}$ mutation operator and improved migration operator of BBO. Our proposed $\mathrm{BBO} / \mathrm{DE} / \mathrm{GEN}$ approach is described as follows.

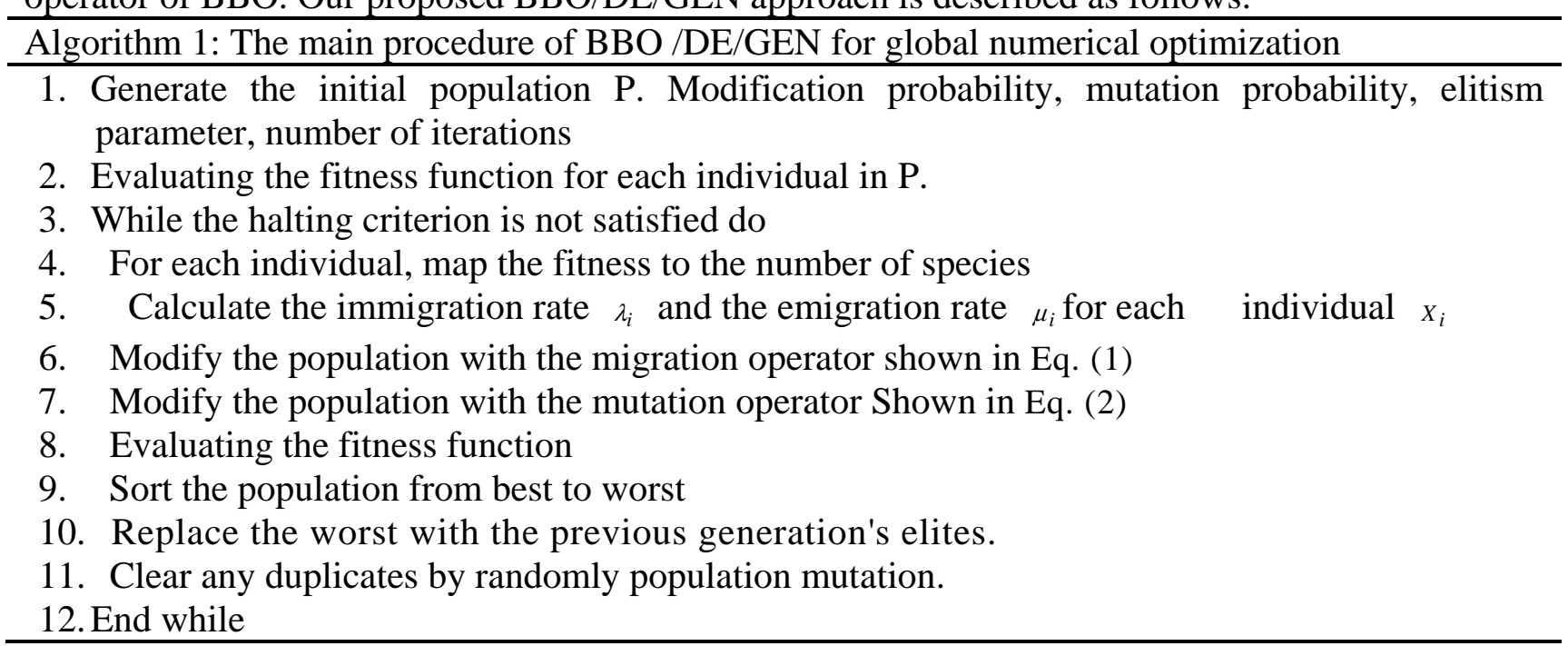

\section{Experiments}

In order to verity the performance of BBO/DE/GEN, 6 benchmark functions are briefly described as follows. Functions f05 is unimodal, functions f09-f13 are multimodal functions where the number of local minima increases exponentially with the problem dimension. They appear to be the most difficult class of problems for many optimization algorithms. Moreover, in our experiments, each function is optimized over 50 independent runs. DE mutation scheme is $\mathrm{DE} / \mathrm{rand} / 1 / \mathrm{bin}$, population size is 50 , generation count is 50 , number of variables in each population is 20 , mutation probability is 0.05 , elitism parameter is 10 , Habitat modification probability is 1 , and scaling factor is 0.5 .

In order to show the superiority of our proposed BBO/DE/GEN approach, we compare it with the $\mathrm{BBO} / \mathrm{DE}$ algorithm and the $\mathrm{BBO}$ algorithm. Minimum cost and Average cost graphs of $\mathrm{BBO} / \mathrm{DE} / \mathrm{GEN}, \mathrm{BBO} / \mathrm{DE}$, and $\mathrm{BBO}$ for 6 benchmark functions are respectively shown in figure 1 to figure 6.

From figure 1 to figure 6 , it is obvious that BBO/DE/GEN performs significantly better than $\mathrm{BBO} / \mathrm{DE}$ and $\mathrm{BBO}$ for 6 typical benchmark functions. 


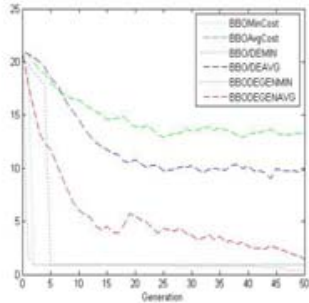

Fig.1. Ackley’s function.

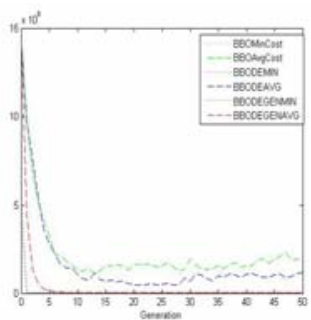

Fig.4. Penalized function 2.

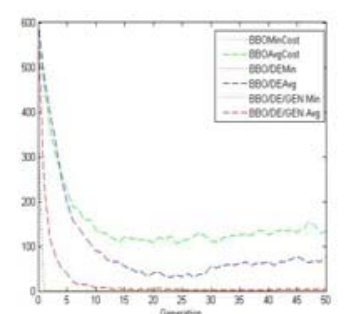

Fig. 2. Griewank function.

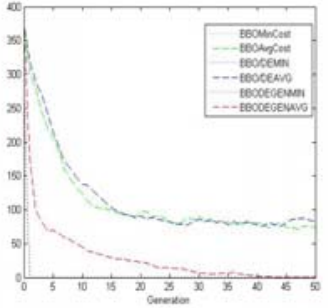

Fig.5. Rastrigin's function.

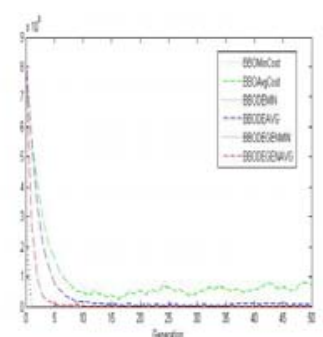

Fig.3. Penalized function 1.

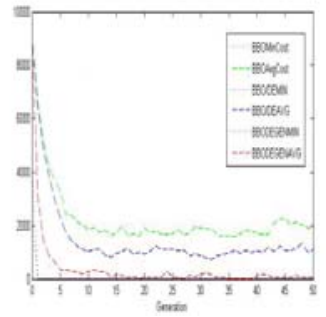

Fig.6. Rosenbrock’s function.

\section{Conclusion}

In order to balance the exploration and the exploitation of $\mathrm{BBO}$, in this paper, we propose a hybrid BBO approach for the global numerical optimization problems, called BBO/DE/GEN, which combines the exploitation of $\mathrm{BBO}$ with the exploration of $\mathrm{DE}$. And a new migration operator is proposed based on number of iteration to improve performance. Since the hybrid mutation operator has a good trade-off between the exploration and the exploitation, it makes our proposed BBO/DE/GEN approach be very effective and efficient. To verify the performance of $\mathrm{BBO} / \mathrm{DE} / \mathrm{GEN}, 6$ typical benchmark functions chosen from literature are employed. Compared with $\mathrm{BBO}, \mathrm{BBO} / \mathrm{DE}$, experimental results show that $\mathrm{BBO} / \mathrm{DE} / \mathrm{GEN}$ is superior to or at least highly competitive with them. It demonstrates the good performance of our approach.

\section{Acknowledgment}

This research was partially supported by the NSFC (61170177).

\section{References}

[1] Storn R, Price K, "Differential evolution-a simple and efficient heuristic for global optimization over continuous spaces”, Global Opt 11(4):341-359, 1997.

[2] Boussaïd, I., Chatterjee, A., Siarry, P., \& Ahmed-Nacer, M., "Hybridizing Biogeography-Based Optimization With Differential Evolution for Optimal Power Allocation in Wireless Sensor Networks”, IEEE TRANSACTIONS ON VEHICULAR TECHNOLOGY, vol. 60, no. 5,pp. 2347-2353, 2011.

[3] Simon, D., “Biogeography-based optimization”, IEEE Trans. Evol. Comput. 12(6), pp.702-713, 2008.

[4] Simon, D., Mehmet, E., Dawei, D., Rick, R, "Markov Models for Biogeography-Based Optimization”, IEEE Transactions on Systems, Man, and Cybernetics-PART B: Cybernetics 41(1), pp.299-306, 2011.

[5] Ma, H., \& Simon, D., "Blended biogeography-based optimization for constrained optimization", Engineering Applications of Artificial Intelligence, 24(3), pp.517-525, 2011. 